\title{
A Corticosteroid-Eluting Sinus Implant Following Endoscopic Sinus Surgery for Chronic Rhinosinusitis: A UK-Based Cost-Effectiveness Analysis
}

\author{
Mehdi Javanbakht ${ }^{1,2}$ (D) $\cdot$ Hesham Saleh $^{3} \cdot$ Mohsen Rezaei Hemami $^{4} \cdot$ Michael Branagan-Harris $^{2} \cdot$ Margaret Boiano $^{2}$
}

Published online: 12 February 2020

(c) The Author(s) 2020

\begin{abstract}
Background Chronic rhinosinusitis (CRS) is one of the commonest chronic health problems among adults in the UK. Around $15 \%$ of CRS patients undergo functional endoscopic sinus surgery (FESS) annually after failing medical treatment. However, as incomplete resolution of symptoms or complications post-operatively is common, the post-operative management is considered to be as important as the surgery itself. A bioabsorbable corticosteroid-eluting sinus implant (CESI) (Propel ${ }^{\circledR}$, mometasone furoate $370 \mu \mathrm{g}$ ) has been used as an alternative post-FESS treatment.

Objective The objective of this study was to assess the cost effectiveness of the corticosteroid-eluting implant versus noncorticosteroid-eluting spacer following FESS for treatment of patients with CRS.

Methods A decision tree model was developed to estimate the cost and effectiveness in each strategy. Costs and effects were estimated from a UK National Health Service (NHS) and personal social services perspective over a 6-month time horizon. Model pathways and parameters were informed by existing clinical guidelines and literature and sensitivity analyses were conducted to explore uncertainties in base-case assumptions.

Results Over a 6-month time horizon, inserting CESI at the end of FESS is less costly ( $£ 4646$ vs. $£ 4655$ per patient) and is the more effective intervention [total quality-adjusted life-years (QALYs) over 6 months 0.443 vs. 0.444] than non-corticosteroid-eluting spacers; hence, it is a dominant strategy. The probabilistic analysis results indicate that CESI following FESS has a $62 \%$ probability of being cost effective at the $£ 20,000$ /per QALY willingness-to-pay threshold and $56 \%$ probability of being a cost-saving intervention.

Conclusions The use of CESI after FESS results in fewer post-operative complications than non-corticosteroid-eluting implants and may be a cost-saving technology over a 6-month time horizon. Although the cost of initial treatment with the CESI is greater, cost savings are made due to a reduction in the number of complications experienced.
\end{abstract}

Electronic supplementary material The online version of this article (https://doi.org/10.1007/s41669-020-00198-8) contains supplementary material, which is available to authorized users.

Mehdi Javanbakht

Javanbakhtm@gmail.com

1 Optimax Access UK Ltd, University of Southampton Science Park, Chilworth, Hampshire, UK

2 Device Access UK Ltd, University of Southampton Science Park, Chilworth, Hampshire, UK

3 Imperial College Healthcare NHS Trust, London, UK

4 PenTAG Health Technology Assessment, University of Exeter, Exeter, UK

\section{Introduction}

Chronic rhinosinusitis (CRS) is one of the commonest chronic health problems among adults in the UK. There are an estimated 250 cases of CRS per 10,000 person-years in an average general practitioner (GP) surgery in the UK [1, 2]. The effect of CRS on quality of life (QoL) is significant when compared with other major illnesses; QoL scores of CRS patients are significantly lower than in other common chronic diseases, such as congestive heart failure, angina, chronic obstructive pulmonary disease, and back pain [3]. Treatment for CRS comprises a trial of optimised medical therapy; surgery, mainly functional endoscopic sinus surgery 


\section{Key Point for Decision Makers}

Inserting a corticosteroid-eluting sinus implant at the end of functional endoscopic sinus surgery is less costly and is the more effective intervention than non-corticosteroid-eluting spacers, and hence it is a dominant strategy.

Results of the probabilistic sensitivity analysis indicate that the Propel ${ }^{\circledR}$ corticosteroid-eluting implant system has over $56 \%$ of likelihood to be cost saving and result in improved patient outcomes.

(FESS), is reserved for recalcitrant cases after the diagnosis is confirmed by radiology and after the failure of medical treatment. Approximately $15 \%$ of patients with CRS undergo FESS annually. There are an estimated 75,000 outpatient consultations annually due to CRS [2]. UK Hospital Episode Statistics (HES) data suggest that approximately 40,000 nose or sinus operations are performed annually in England and Wales [2].

Post-operative treatment regimens are considered to be as important as the surgery itself $[4,5]$. Common causes of suboptimal post-operative results include recurrent inflammation, polyposis, adhesion/synechiae formation, middle turbinate (MT) lateralization and stenosis of sinus ostia [6]. Post-operative interventions, which are aimed at minimising these issues, include medical therapy and debridement to lyse adhesions. These interventions are time-consuming and costly and negatively impact on patients' QoL [7-10].

Corticosteroids are effective in minimising post-operative complications but current post-operative medical therapies have significant limitations. Oral corticosteroids carry systemic risks, including mood changes, calcium demineralisation, cataract formation and, rarely, aseptic necrosis of the femoral head [11]. The efficacy of topical corticosteroid spray in this setting is limited by post-operative edema, discharge, crusting and poor patient compliance. A bioabsorbable corticosteroid-eluting sinus implant (CESI) (Propel ${ }^{\circledR}$, mometasone furoate $370 \mu \mathrm{g}$; Intersect ENT, Inc., Menlo Park, CA, USA) has been shown to be safe and effective in maintaining patency of the ethmoid sinus, thereby reducing the need for post-operative interventions, including surgical adhesion lysis and/or the use of oral corticosteroids. The CESI technology separates mucosal tissues, provides stabilisation of the MT, prevents obstruction by adhesions and, due to its corticosteroid-elution properties, reduces edema during the post-operative healing period $[5,12,13]$. A metaanalysis by Han et al. [14] showed that Propel ${ }^{\circledR}$ can significantly reduce the need for intervention after FESS, including surgical procedures and oral corticosteroids, through localised, controlled delivery of mometasone furoate over
30 days. Propel ${ }^{\circledR}$ is effective in these ways by (1) maintaining patency (like a spacer); and (2) delivering corticosteroids without the need for patient interaction, which improves compliance.

With more than 40,000 sinus surgery procedures performed in the UK every year (NHS digital 2018-17), it is important to know how the implementation of novel CRSrelated interventions will affect the overall efficacy of postoperative care. Although application of corticosteroid-eluting bioabsorbable stents during endoscopic sinus surgery has been recommended by the National Institute for Health and Care Excellence (NICE) (Interventional Procedures Guidance IPG551) [15], to date, a rigorous economic analysis of the costs and consequence of FESS plus CESI versus FESS plus non-corticosteroid-eluting spacer for CRS has not been performed in the UK. The objective of this study is to evaluate the cost effectiveness of the Propel ${ }^{\circledR}$ mometasone CESI following FESS compared with a non-corticosteroideluting spacer following FESS for the treatment of patients with CRS.

\section{Methods}

\subsection{Model Overview}

A de novo decision-analytic model was developed using Microsoft Excel ${ }^{\circledR}$ (Microsoft Corp., Redmond, WA, USA) (Fig. 1). CRS patients were not systematically engaged to validate the model; however, one of our research team members (Margaret Boiano) has extensive research experience in CRS surgery and she was engaged in developing the model from the beginning. Additionally, the model was validated by several clinicians involved in treating patients undergoing CRS surgery. In order to capture the costs and benefits of the intervention and comparator, a 6-month time horizon was adopted in the analysis. We used a 6-month time horizon due to a lack of long-term evidence. Additionally, it is not expected that Propel ${ }^{\circledR}$ will have a long-term impact on the incidence of post-operative complications as it will be dissolved after 2-3 months.

The model was used to simulate the clinical management of a CRS patient undergoing surgery and receiving one of two treatment strategies: (1) an intervention consisting of FESS plus CESIs bilaterally (Propel ${ }^{\circledR}$ Sinus Implant, Intersect ENT, Inc., Menlo Park, CA, USA) (intervention); or (2) FESS plus two non-corticosteroid-eluting spacers (comparator). Since application of a spacer or non-drug eluting stent has been recommended by NICE (see Guidance IPG551 [15]), this reflects the current treatment pathway in the England. In both treatment strategies, there were three potential post-operative complications that would require clinical intervention: (1) recurrent polyposis requiring oral 


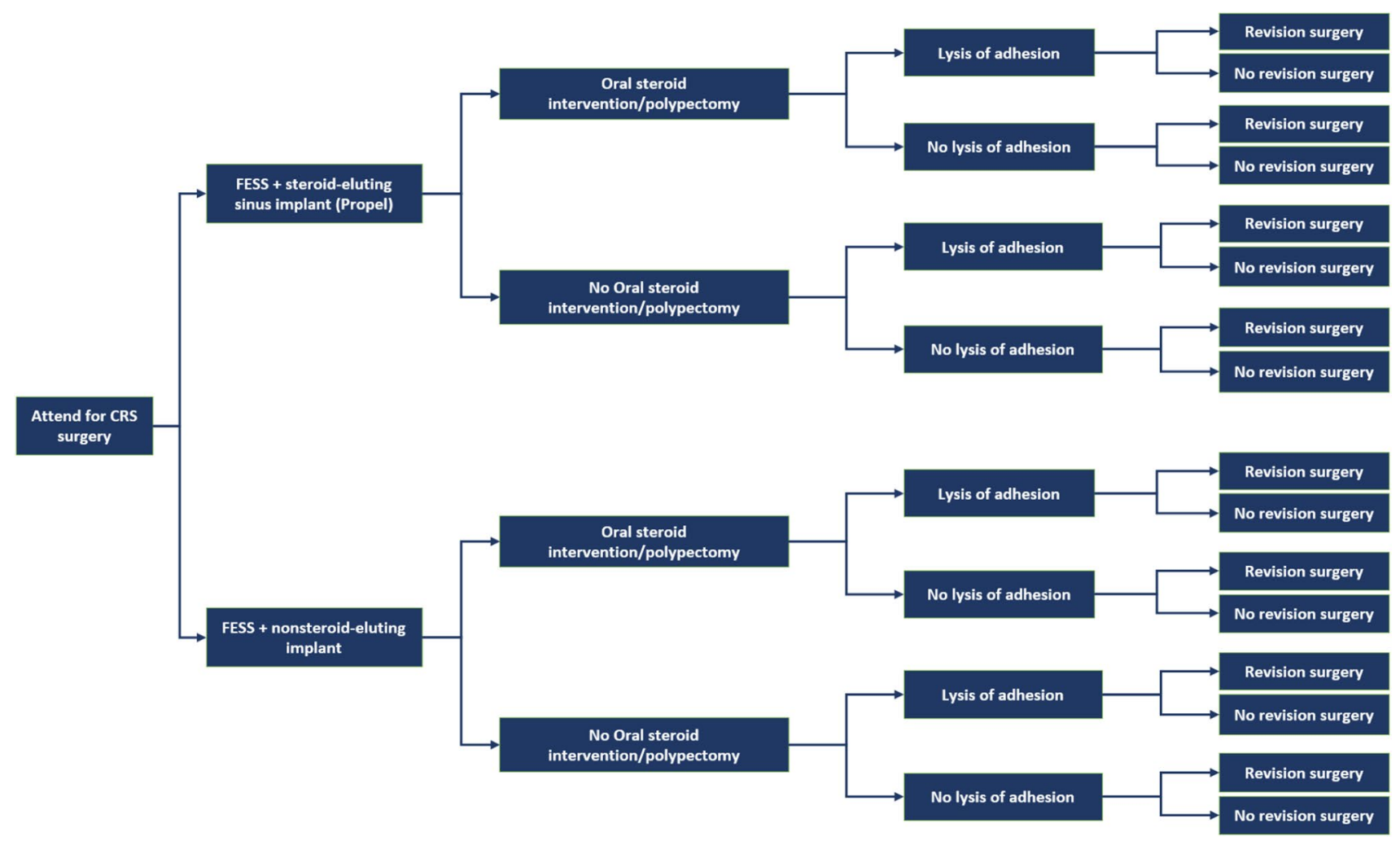

Fig. 1 Decision tree to assess cost effectiveness of corticosteroid-eluting sinus implant versus non-corticosteroid-eluting spacer following functional endoscopic sinus surgery (FESS) for the treatments of patients with chronic rhinosinusitis (CRS)

corticosteroids and surgical treatment (i.e. polypectomy); (2) severe adhesions requiring lysis; or (3) a revision FESS. The following sections describe the data used in the economic model, including information on the clinical effectiveness, costs, and quality-of-life relating to the different treatment strategies.

\subsection{Clinical Effectiveness}

The results from the meta-analysis conducted by Han et al. [14] were used to inform the values for post-operative complications for each strategy during the first 2 months following FESS. This meta-analysis was based on the results from two randomised clinical trials (RCTs) $[12,13]$ that were conducted in the USA. Due to a lack of evidence about the incidence of post-operative complications in the UK, it was assumed that the rates in the comparator arm are the same as those in the control group in the aforementioned RCTs. The post-operative complications are prevalent and this is supported by a recently conducted review [16]. In the previous NICE health technology assessment (HTA) submission [17], it was assumed that at least $41 \%$ of patients will have one to two GP visits within the first 3 months following surgery (the mean number of GP visits was 1.86). It was also assumed that the monthly rate of GP visits after the first
3 months was 0.12 in the previous NICE submission [17]. In the current study it was assumed that if procedures are required to treat the complications, it will be conducted by an ear-nose-throat (ENT) specialist, not a GP.

Based on the results from the same meta-analysis it was assumed that rates of lysis of adhesions within the first 2 months post-FESS were $25.2 \%$ and $14.2 \%$ in the control and intervention groups, respectively. The rate of polyposis grade 2-3, which requires oral corticosteroid intervention or polypectomy, were assumed to be $38.5 \%$ and $22 \%$, respectively (Table 1). Beyond 3 months, patients may experience recurrent polyposis requiring oral corticosteroids and surgical treatment (polypectomy and/or revision FESS). The polyposis recurrence rates more than 3 months postoperatively were obtained from a study conducted by Rizzo et al. [18]. The authors of this study conducted imputation methods to estimate the incidence rates of post-operative complications up to 6 months post-surgery. In the base-case analysis, it was assumed that the need for adhesion lysis was zero after the first 3 months post-operatively.

The revision surgery rates for each intervention were obtained from the ADVANCE study [5], an open-label prospective, multicentre clinical study that was conducted in the USA. It was assumed that patients requiring revision surgery would receive FESS alone. Mortality was not included in the 
Table 1 Clinical inputs

\begin{tabular}{|c|c|c|c|c|c|c|c|c|c|}
\hline \multirow{2}{*}{$\begin{array}{l}\text { Time point } \\
\text { post-FESS } \\
\text { (months) }\end{array}$} & \multirow[t]{2}{*}{ Outcomes } & \multicolumn{3}{|c|}{ FESS + CESI $(\%)$} & \multicolumn{3}{|c|}{$\begin{array}{l}\text { FESS + non-corticosteroid- } \\
\text { eluting spacer }(\%)\end{array}$} & \multirow[t]{2}{*}{ Distribution } & \multirow[t]{2}{*}{ References } \\
\hline & & Mean & Lower limit & Upper limit & Mean & Lower limit & Upper limit & & \\
\hline \multirow[t]{3}{*}{$1-2$} & Lysis of adhesions & 14.2 & 8.8 & 21.3 & 25.2 & 2.7 & 85.0 & Beta & {$[14,18]$} \\
\hline & Polyposis recurrence & 22.1 & 14.9 & 30.9 & 38.5 & 35.0 & 42.0 & Beta & {$[14,18]$} \\
\hline & Nasal corticosteroid spray use & 0.0 & NA & NA & 100.0 & NA & NA & Beta & {$[14,18]$} \\
\hline \multirow[t]{2}{*}{$3-5$} & Lysis of adhesions & 0.0 & NA & NA & 27.6 & 4.6 & 60.0 & Beta & {$[14,18]$} \\
\hline & Polyposis recurrence & 14.6 & 7.3 & 21.9 & 31.0 & 27.0 & 35.0 & Beta & {$[14,18]$} \\
\hline \multirow[t]{3}{*}{6} & Revision surgery & 2.2 & NA & NA & 4.7 & NA & NA & Beta & {$[5]$} \\
\hline & Lysis of adhesions & 0.0 & NA & NA & 39.8 & 19.9 & 59.7 & Beta & {$[14,18]$} \\
\hline & Polyposis recurrence & 7.1 & 3.6 & 10.7 & 23.5 & 11.8 & 35.3 & Beta & {$[14,18]$} \\
\hline
\end{tabular}

CESI corticosteroid-eluting sinus implant, FESS functional endoscopic sinus surgery, NA not available

model as CRS-related mortality is very rare and not expected to differ among interventions. The clinical effectiveness data used in the model are shown in Table 1.

\subsection{Resource Use and Costs}

All costs were estimated from a UK National Health Service (NHS) and Personal Social Services perspective. The following costs were included: cost of initial surgery, device cost (e.g. Propel ${ }^{\circledR}$ CESI or non-corticosteroid-eluting spacer), treatment costs of post-operative adverse events, cost of revision surgery and cost of medication. Based on clinical expert opinion and the hospital episode data it was assumed that all lysis of adhesions procedures would be conducted as outpatient procedures. Those patients who developed polyposis were treated using medication and a polypectomy procedure and/or revision sinus surgery. Unit costs for these components of resource use were obtained from the literature or obtained through other relevant sources, such as NHS reference costs [19], Personal Social Services Research Unit (PSSRU) unit costs [20], British National Formulary (BNF) data [21] and/ or manufacturer price lists. Costs were measured in British Pound Sterling ( $($ ) for the year 2018 and are shown in Table 2. Where required, costs were inflated to this price year using the hospital and community health services (HCHS) index listed in the Unit Costs of Health and Social Care 2018 [20].

\subsection{Utilities}

The utility scores included in the model are a measure of the QoL associated with either a successful or a failed surgery. Due to a lack of UK-based evidence, utility values

Table 2 Unit costs used in the model

\begin{tabular}{|c|c|c|c|}
\hline Cost & Value $(£)$ & Distribution & Source \\
\hline \multicolumn{4}{|l|}{ Procedure cost } \\
\hline Cost of procedure: FESS & 2537 & Gamma & $\begin{array}{l}\text { NHS reference cost-intermediate sinus procedures-CA28Z-elective inpatient } \\
\text { [19] }\end{array}$ \\
\hline \multicolumn{4}{|l|}{ Device cost } \\
\hline Cost of Propel $®$ device & 580 & Fixed & Intersect $^{\circledR}$ ENT [24] \\
\hline $\begin{array}{l}\text { Non-drug-eluting sinus } \\
\text { spacer (Price for one pair) }\end{array}$ & 62.5 & Fixed & NasoPore [25] \\
\hline \multicolumn{4}{|l|}{ Post-operative treatment costs } \\
\hline Unit cost of lysis of adhesion & 149 & Gamma & NHS reference cost 2018 —CA24A — lysis of adhesion [19] \\
\hline Unit cost of nasal polypectomy & 1739 & Gamma & NHS reference cost 2018_CA14Z_-polypectomy [19] \\
\hline Cost of revision surgery: FESS & 2263 & Gamma & $\begin{array}{l}\text { NHS reference cost-intermediate sinus procedures-CA28_-elective inpatient } \\
{[19]}\end{array}$ \\
\hline \multicolumn{4}{|l|}{ Medications and visits } \\
\hline $\begin{array}{l}\text { Cost of medication (corticos- } \\
\text { teroid nasal spray + antibiot- } \\
\text { ics) }\end{array}$ & 26 & Fixed & $\begin{array}{l}\text { BNF corticosteroid nasal spray-fluticasone propionate + macrolide-azithromycin } \\
500 \mathrm{mg} \text { once daily for } 3 \text { days [21] }\end{array}$ \\
\hline Cost of ENT visit & 96 & Gamma & NHS reference cost 2018 — consultant led [19] \\
\hline
\end{tabular}

$B N F$ British National Formulary, ENT ear-nose-throat, FESS functional endoscopic sinus surgery, NHS National Health Service 
were all derived from a study by Remenschneider et al. [22], which was conducted in the USA using the EQ-5D tool. Disutility associated with post-operative complications was not included, as they could be treated and resolved quickly. Utility values are shown in Table 3. A successful surgery was defined as a surgery without need for revision. For those who needed revision surgery, the utility value was assumed to be the same as the utility value of the pre-FESS health state. The quality-adjusted life-years (QALYs) were generated using the area under the curve method.

\subsection{Analysis}

The cumulative estimates of costs and consequences are reported for both strategies. Probabilistic and deterministic sensitivity analyses were conducted to explore parameter and other forms of uncertainty surrounding the estimates of cost consequence. Deterministic sensitivity analyses were conducted to investigate the impact of varying key assumptions and/or parameter values used in the base-case analysis. The model also incorporated probabilistic sensitivity analysis (PSA) to quantify parameter uncertainty. To conduct the PSA, probabilistic distributions were assigned to each input in the model (these are presented in Tables 1,2,3) and were used to randomly select new plausible values. Each new sampled value was applied in the model and the new results of the model were recorded. This process was repeated for a large number of iterations $(10,000)$ to produce a distribution of results from the model.

\section{Results}

A Monte Carlo simulation (10,000 samples) was performed to obtain probabilistic estimates of the cost effectiveness of CESI following FESS compared with a non-corticosteroideluting spacer following FESS. Over a 6-month time horizon, CESI following FESS is less costly ( $£ 4646$ vs. $£ 4655$ per patient) and is the more effective strategy ( 0.444 vs. 0.443 QALYs), hence it is a dominant strategy (Table 4).

The cost-effectiveness acceptability curve (CEAC) shown in Fig. 2 indicates that, over a 6-month time horizon, the CESI following FESS strategy has a moderate probability of being cost effective: $62 \%$ at a willingness to pay (WTP) of $£ 20,000$ and $66 \%$ at a $£ 30,000$ WTP. Total aggregated costs, shown in Table 5, which do not consider utility values, indicate that total costs are lower for the intervention than for the comparator. The overall population that could benefit from this intervention in the UK is estimated to be 16,693 (Electronic Supplementary Material Table S1). The total annual number of post-operative procedures in the UK is reported in Electronic Supplementary Material Table S2. The overall costs in the two strategies are reported in Table 5. Although the cost of initial treatment with CESI is greater, cost savings are derived from fewer occurrences of symptom recurrence/surgical failure, resulting in an overall cost saving of $£ 160,692$ in the UK over a 6-month time horizon. Furthermore, results from the PSA showed that the CESI arm has a likelihood of over $56 \%$ of being a cost-saving strategy. The results from the deterministic sensitivity analyses showed that post-operative intervention rates and the probability of receiving a polypectomy procedure for polyposis were
Table 3 Utility values included in the model

\begin{tabular}{lllll}
\hline Event/health state & Baseline value & SE & Distribution & References \\
\hline Utility pre-FESS & 0.81 & 0.13 & Beta & {$[22]$} \\
Utility post-successful FESS & 0.89 & 0.10 & Beta & {$[22]$} \\
\hline
\end{tabular}

FESS functional endoscopic sinus surgery, SE standard error

Table 4 Base-case probabilistic results over a 6-month time horizon

\begin{tabular}{|c|c|c|c|c|c|c|c|}
\hline \multirow[t]{2}{*}{ Strategy } & \multirow[t]{2}{*}{ 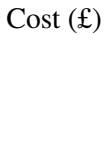 } & \multirow[t]{2}{*}{$\begin{array}{l}\text { Incremental } \\
\text { cost }(\mathfrak{f})\end{array}$} & \multirow[t]{2}{*}{ QALY } & \multirow[t]{2}{*}{$\begin{array}{l}\text { Incremental } \\
\text { QALY }\end{array}$} & \multirow[t]{2}{*}{ 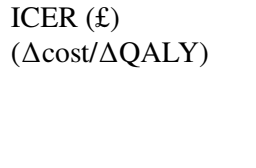 } & \multicolumn{2}{|c|}{$\begin{array}{l}\text { Probability cost } \\
\text { effective for different } \\
\text { WTP }(\%)\end{array}$} \\
\hline & & & & & & $£ 20,000$ & $£ 30,000$ \\
\hline FESS + CESI $\left(\right.$ Propel $\left.^{\circledR}\right)$ (intervention) & 4646 & -10 & 0.444 & 0.0010 & $\begin{array}{l}\text { FESS + CESI } \\
\left(\text { Propel }^{\circledR}\right) \text { is domi- } \\
\text { nant }\end{array}$ & 62 & 66 \\
\hline $\begin{array}{l}\text { FESS + non-corticosteroid-eluting spacer } \\
\text { (comparator) }\end{array}$ & 4655 & & 0.443 & & & 38 & 34 \\
\hline
\end{tabular}

$C E S I$ corticosteroid-eluting sinus implant, FESS functional endoscopic sinus surgery, ICER incremental cost-effectiveness ratio, $Q A L Y$ qualityadjusted life-year, WTP willingness to pay threshold 

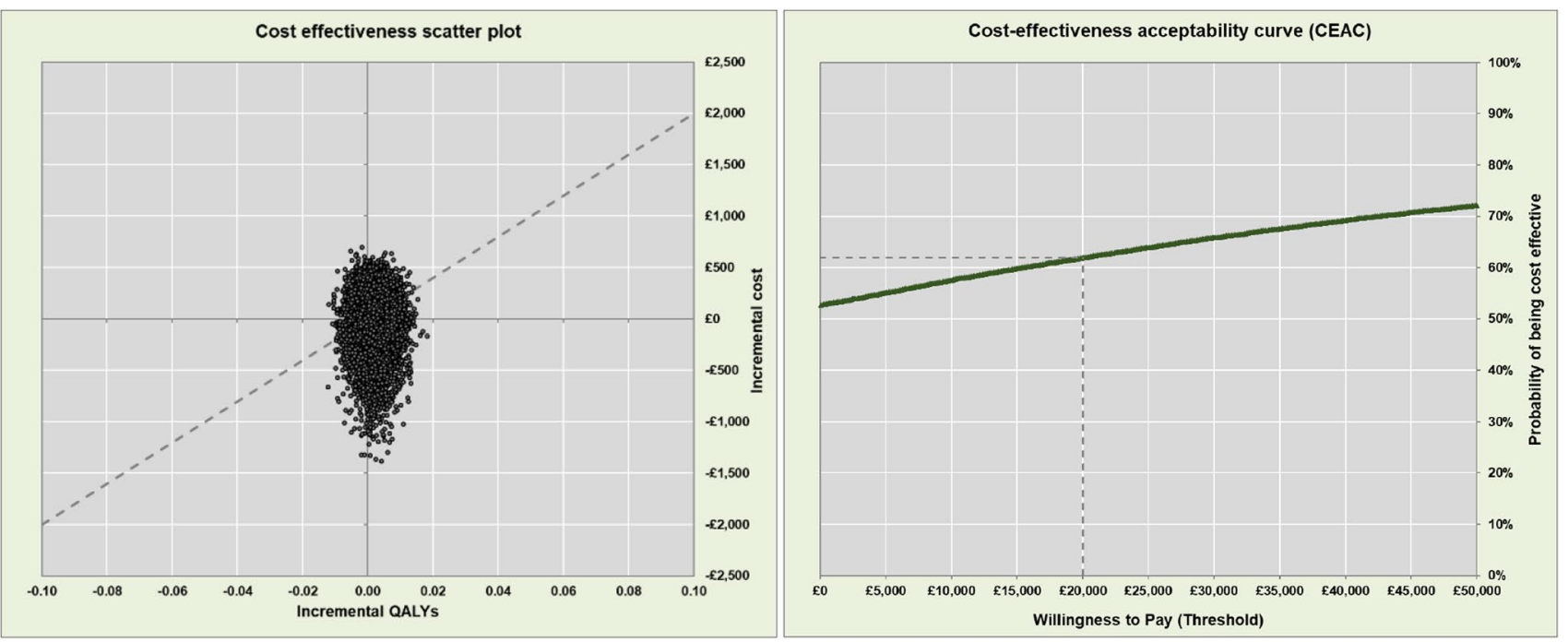

Fig. 2 Cost-effectiveness scatter plot and cost-effectiveness acceptability curve (CEAC)

Table 5 Total aggregated costs and effectiveness in the UK for 16,693 patients with chronic rhinosinusitis

\begin{tabular}{lccr}
\hline Costs & $\begin{array}{l}\text { FESS + CESI (inter- } \\
\text { vention) }\end{array}$ & $\begin{array}{l}\text { FESS + non-corticosteroid-eluting } \\
\text { spacer (comparator) }\end{array}$ & Difference \\
\hline Initial treatment & $61,710,402$ & $43,573,570$ & $18,136,832$ \\
Polyposis & $14,504,939$ & $30,798,158$ & $-16,293,219$ \\
Lysis of adhesion & 352,958 & $1,250,764$ & $-897,806$ \\
Revision surgery & 981,571 & $2,088,070$ & $-1,106,499$ \\
Total & $77,549,871$ & $77,710,563$ & $-160,692$ \\
\hline
\end{tabular}

CESI corticosteroid-eluting sinus implant, FESS functional endoscopic sinus surgery

Table 6 Results from deterministic sensitivity analysis

\begin{tabular}{|c|c|c|c|c|c|c|c|}
\hline \multirow[t]{2}{*}{ Strategy } & \multirow[t]{2}{*}{ Cost $(\mathfrak{E})$} & \multirow[t]{2}{*}{ 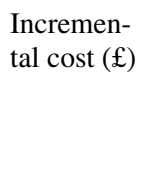 } & \multirow[t]{2}{*}{ QALY } & \multirow[t]{2}{*}{$\begin{array}{l}\text { Incremen- } \\
\text { tal QALY }\end{array}$} & \multirow[t]{2}{*}{$\begin{array}{l}\operatorname{ICER}(\mathfrak{f}) \\
(\Delta \cos t / \Delta \mathrm{QALY})\end{array}$} & \multicolumn{2}{|c|}{$\begin{array}{l}\text { Probability cos } \\
\text { effective for di } \\
\text { ent WTP }(\%)\end{array}$} \\
\hline & & & & & & $£ 20,000$ & $£ 30$, \\
\hline \multicolumn{8}{|c|}{ Sensitivity analysis 1 : informing the post-operative intervention rates from Han et al. [14], meaning no treatment effect beyond 3 months } \\
\hline FESS + CESI (intervention) & 4215 & 655 & 0.2216 & 0.0005 & $1,320,824$ & 29 & 29 \\
\hline FESS + non-corticosteroid-eluting spacer (comparator) & 3560 & & 0.2221 & & & 71 & 71 \\
\hline
\end{tabular}

Sensitivity analysis 2 : assuming $50 \%$ of patients with polyposis would receive polypectomy procedure and the rest would be treated with oral corticosteroids only

$\begin{array}{lllllll}\text { FESS + CESI (intervention) } & 4048 & 662 & 0.444 & 0.0010 & 667,414 & 29 \\ \text { FESS + non-corticosteroid-eluting spacer (comparator) } & 3386 & & 0.443 & & 71 & 71\end{array}$

Sensitivity analysis 3: informing the post-operative intervention rates from Han et al. [14], meaning no treatment effect beyond 3 months, and also assuming $50 \%$ of patients with polyposis would receive polypectomy procedure and the rest would be treated with oral corticosteroids only

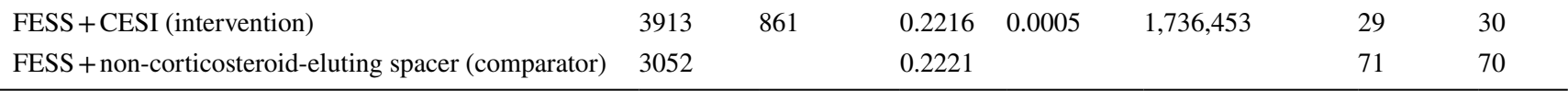

CESI corticosteroid-eluting sinus implant, FESS functional endoscopic sinus surgery, $Q A L Y$ quality-adjusted life-year, ICER incremental costeffectiveness ratio, WTP willingness-to-pay threshold 
among the main drivers of the results (Table 6). We could not identify any sources to estimate the uncertainty around the revision rate. In order to address this we conducted a scenario analysis in which we estimated the uncertainty around the revision rate by multiplying the mean revision rate by $\pm 25 \%$ to estimate the upper and lower limits. Results of this scenario analysis indicated that the overall conclusion is still the same, meaning that CESI following FESS has a $62 \%$ probability of being cost effective at the $£ 20,000 \mathrm{WTP}$ threshold (Electronic Supplementary Material Fig. S1).

\section{Discussion}

Results of this study indicate that, on a per-patient basis, the strategy of CESI following FESS is marginally less costly than the comparator and produces more QALYs with a significant probability of being cost effective $(62 \%$ and $66 \%$ at WTP $£ 20,000$ and $£ 30,000$, respectively) over a 6-month period. The CEAC produced also shows that the probability of being cost effective increases for the intervention as the WTP increases.

Results of this study are largely consistent with a previous US-based cost-effectiveness analysis comparing a mometasone furoate CESI following FESS with a non-corticosteroid-eluting spacer for patients with refractory CRS [23]. In this US-based study, the CESI strategy cost more (\$US1573 vs. \$US365) but resulted in an incremental costeffectiveness ratio (ICER) of \$US5490 per post-operative intervention avoided within 60 days after FESS. Although the primary endpoint of our analysis differs from that in this US-based analysis, our findings are consistent with the results presented here.

As with all modelling studies, several limitations exist in this analysis that must be considered when interpreting the results. Firstly, data to inform decrements in QoL associated with adverse events were unavailable so were omitted from the analysis. Therefore, the overall QALYs reported for each strategy may be an over-estimate. However, including decrements would likely have increased the cost effectiveness of the CESI strategy because the intervention results in lower complication rates over a 6 -month time horizon. There was also a lack of information available on longer-term clinical effectiveness of the two treatment strategies after 6 months. The meta-analysis from which clinical data were drawn only included effectiveness data over a 3-month time horizon and effectiveness beyond 3 months has been extrapolated by Rizzo et al. [18]. Although uncertainty was present in the model results because of these data limitations, this was addressed by applying probability distributions to parameters, where possible, and exploring parameter variation in sensitivity analyses.
The major strength of this analysis is that it is, as far as we are aware, the first economic model to explore the relative cost effectiveness of these two treatment strategies following FESS in a UK setting. Findings from this analysis may be used to assist in decision-making regarding the most appropriate treatment to use for patients with CRS post-operatively. Finally, the analysis was conducted using best practice methods and a range of sensitivity analyses were conducted to account for uncertainty.

\section{Conclusion}

Treatment with FESS plus CESI provides better outcomes and may be cost-saving compared with FESS plus non-corticosteroid-eluting spacers over a 6-month horizon. CESI may be a suitable alternative for post-FESS treatment. Although the cost of initial treatment with CESI is higher, cost savings accrue due to a reduction in the number of adverse events experienced.

Author contributions $\mathrm{MJ}$ and $\mathrm{MRH}$ were responsible for developing and populating the economic model and drafting the first version of the manuscript. All authors provided inputs for the model and read and approved the final draft of the manuscript.

Data Availability Statement The authors declare that all of the data supporting the findings of this study are available within the article (or the Electronic Supplementary Material of the article).

\section{Compliance with Ethical Standards}

Funding This report is independent research funded by Intersect ENT Corporation.

Conflict of Interest HS has no conflicts of interest that are directly relevant to the content of this article. Device Access (MJ, MRH, MB and $\mathrm{MBH}$ ) received funds from Intersect ENT Corporation during the conduct of the study.

Open Access This article is licensed under a Creative Commons Attribution-NonCommercial 4.0 International License, which permits any non-commercial use, sharing, adaptation, distribution and reproduction in any medium or format, as long as you give appropriate credit to the original author(s) and the source, provide a link to the Creative Commons licence, and indicate if changes were made. The images or other third party material in this article are included in the article's Creative Commons licence, unless indicated otherwise in a credit line to the material. If material is not included in the article's Creative Commons licence and your intended use is not permitted by statutory regulation or exceeds the permitted use, you will need to obtain permission directly from the copyright holder.To view a copy of this licence, visit http://creativecommons.org/licenses/by-nc/4.0/. 


\section{References}

1. Henderson R. Sinusitis. 2019. https://patient.info/doctor/sinusitispro. Accessed Sept 2019.

2. Philpott C, Hopkins C, Erskine S, Kumar N, Robertson A, Farboud A, et al. The burden of revision sinonasal surgery in the UK - data from the chronic rhinosinusitis epidemiology Study (CRES): a cross-sectional study. BMJ Open. 2015;5(4):e006680.

3. Metson RB, Gliklich RE. Clinical outcomes in patients with chronic sinusitis. Laryngoscope. 2000;110(3 Pt 3):24-8.

4. Eloy P, Andrews P, Poirrier AL. Postoperative care in endoscopic sinus surgery: a critical review. Curr Opin Otolaryngol Head Neck Surg. 2017;25(1):35-42.

5. Forwith KD, Chandra RK, Yun PT, Miller SK, Jampel HD. ADVANCE: a multisite trial of bioabsorbable steroid-eluting sinus implants. Laryngoscope. 2011;121(11):2473-80.

6. Chaaban MR, Rana N, Baillargeon J, Baillargeon G, Resto V, Kuo YF. Outcomes and complications of balloon and conventional functional endoscopic sinus surgery. Am J Rhinol Allergy. 2018;32(5):388-96.

7. Philpott $\mathrm{CH}$, Erskine C, Kumar S, Robertson N, Farboud A, Ahmed A, et al. The burden of revision sinonasal surgery in the UK-data from the chronic rhinosinusitis epidemiology Study (CRES): a cross-sectional study. BMJ Open. 2015;5(4):e006680.

8. Al Badaai Y, Valdes CJ, Samaha M. Outcomes and cost benefits of functional endoscopic sinus surgery in severely asthmatic patients with chronic rhinosinusitis. J Laryngol Otol. 2014;128(6):512-7.

9. Sahlstrand-Johnson P, Hopkins C, Ohlsson B, Ahlner-Elmqvist $\mathrm{M}$. The effect of endoscopic sinus surgery on quality of life and absenteeism in patients with chronic rhinosinuitis - a multi-centre study. Rhinology. 2017;55(3):251-61.

10. Saedi B, Sadeghi M, Akhavan-Khaleghi N, Seifmanesh H. Impact of endoscopic sinus surgery on the quality of life of patients with nasal polyposis. B-ENT. 2014;10(1):59-655.

11. Lipworth BJ. Systemic adverse effects of inhaled corticosteroid therapy: a systematic review and meta-analysis. Arch Intern Med. 1999;159(9):941-55.

12. Marple BF, Smith TL, Han JK, Gould AR, Jampel HD, Stambaugh JW, et al. Advance II: a prospective, randomized study assessing safety and efficacy of bioabsorbable steroid-releasing sinus implants. Otolaryngol Head Neck Surg. 2012;146(6):1004-111.
13. Murr AH, Smith TL, Hwang PH, Bhattacharyya N, Lanier BJ, Stambaugh JW, et al. Safety and efficacy of a novel bioabsorbable, steroid-eluting sinus stent. Int Forum Allergy Rhinol. 2011;1(1):23-322.

14. Han JK, Marple BF, Smith TL, Murr AH, Lanier BJ, Stambaugh $\mathrm{JW}$, et al. Effect of steroid-releasing sinus implants on postoperative medical and surgical interventions: an efficacy meta-analysis. Int Forum Allergy Rhinol. 2012;2(4):271-9.

15. NICE. Corticosteroid-eluting bioabsorbable stent or spacer insertion during endoscopic sinus surgery to treat chronic rhinosinusitis. Interventional procedures guidance IPG551. London: Nice; 2016. https://www.nice.org.uk/guidance/ipg551. Accessed Sept 2019.

16. Noon E, Hopkins C. Review article: outcomes in endoscopic sinus surgery. BMC Ear Nose Throat Disord. 2016;16(1):9.

17. Jenks M, Willits I, Turner EE, Hewitt N, Arber M, Cole H, et al. The XprESS multi-sinus dilation system for the treatment of chronic sinusitis: a NICE medical technology guidance. Appl Health Econ Health Policy. 2017;15(5):567-82.

18. Rizzo JA, Rudmik L, Mallow PJ, Palli SR. Budget impact analysis of bioabsorbable drug-eluting sinus implants following endoscopic sinus surgery. J Med Econ. 2016;19(9):829-35.

19. Department of Health. Reference costs 2017-18. 2018.

20. Burns LCA. Unit costs of health and social care 2018. Canterbury: Personal Social Services Research Unit, University of Kent; 2018. https://doi.org/10.22024/UniKent/01.02.70995.

21. British National Formulary. BNF Online. https://www.medicinesc omplete.com $/ \mathrm{mc} /$ ?utm_source=bnforg\&utm_medium $=$ homep age\&utm_campaign=medicinescomplete. Accessed Sept 2019.

22. Remenschneider AK, Scangas G, Meier JC, Gray ST, Holbrook $\mathrm{EH}$, Gliklich RE, et al. EQ-5D-derived health utility values in patients undergoing surgery for chronic rhinosinusitis. Laryngoscope. 2015;125(5):1056-61.

23. Rudmik L, Smith TL. Economic evaluation of a steroid-eluting sinus implant following endoscopic sinus surgery for chronic rhinosinusitis. Otolaryngol Head Neck Surg. 2014;151(2):359-66.

24. Intersect ${ }^{\circledR}$ ENT. PROPEL ${ }^{\circledR}$ sinus implants. 2018. https://www. intersectent.com/technologies/. Accessed Sept 2019.

25. Stryker. NasoPore bioresorbable nasal dressing. 2018. https:// www.stryker.com/content/stryker/us/en/nse/products/nasoporebioresorbable-nasal-dressing.html. Accessed Sept 2019. 